\title{
Emploi modal de proposer que $p$ en contexte non-volitif
}

\author{
Hugues ENGEL \\ Mats FORSGREN \\ Françoise SULLET-NYLANDER \\ Université de Stockholm, Suède
}

\begin{abstract}
«En se risquant dans ce domaine [sc celui de la variation modale] difficilement accessible, l'étranger risque fort de se tromper, surtout s'il a la prétention d'expliquer plutôt que de constater. » (Nordahl 1969)
\end{abstract}

« Avant toute chose, il faut cependant mettre en garde contre l'illusion d'une prédictibilité rigoureuse. L'emploi du subjonctif obéit à des tendances beaucoup plus qu'à des règles, et, ainsi, les conceptions rigides se vouent elles-mêmes à l'échec. Ce que montrent les descriptions les plus fines, c'est l'importante fréquence de l'alternance modale : alternances significatives [...] ou alternances plus ou moins libres ». (Martin 1983)

\section{Introduction : le subjonctif en linguistique française}

La variation modale du verbe français (et, plus généralement, du verbe roman) a été traitée de façon spécialisée surtout par des non-Français. Bien plus, comme l'écrit le Danois Gerhard Boysen, « [1]e goût des études sur le subjonctif semble s'être particulièrement bien développé en Scandinavie » (Boysen 1971 : 263). L'explication en est patente : puisque le subjonctif n'existe pratiquement plus dans les langues scandinaves ${ }^{1}$, sa maîtrise pose des problèmes assez redoutables pour les apprenants de français langue étrangère. C'est aussi pourquoi les grammaires scandinaves de français L2 consacrent généralement bon nombre de pages à des exposés extensifs des différents contextes où apparaît le subjonctif. Les ouvrages spécialisés de souche scandinave, se conformant là à une tradition résolument empiricopositiviste et descriptiviste, se basent en général sur de vastes recueils de données authentiques, exploités de façon inductive et sans idées préconçues, dans le but de détecter des corrélations entre critères formels (morphosyntaxiques) et variation modale, corrélations jugées nécessaires pour

$1 \quad$ Il n'en reste plus, par exemple en suédois, que dans quelques expressions figées du type «Leve konungen! » («Vive le roi !»).

(C) Cahiers Chronos 21 (2010) : 85-101. 
pouvoir passer ultérieurement à d'éventuelles hypothèses explicatives ${ }^{2}$. Ainsi Börjesson a-t-il consacré une étude au mode de la complétive (Börjesson $1966^{3}$ ), qui a été suivie de l'énorme enquête de Nordahl, également sur la complétive et basée sur non moins de 407 textes littéraires, complétés par un nombre élevé de journaux, ce qui avait donné, chiffre plus que respectable pour l'époque "précomputationnelle», environ 24000 occurrences (Nordahl 1969). C'était, en effet, de la linguistique de corpus bien avant l'avènement du terme dans son acception moderne. Ajoutons Boysen (1971) et plusieurs études uppsaliennes sur le mode de la relative française ou romane (Carlsson 1969, 1973, 1974 ; Eriksson 1979). La grande Grammaire française de Togeby (Togeby 1982) constitue également une mine d'exemples authentiques ${ }^{4}$. Citons enfin, pour la tradition scandinave, l'étude de Nølke (1985), sur laquelle nous aurons l'occasion de revenir par la suite.

En revanche, l'intérêt des grammairiens et linguistes français s'est de tout temps porté, avant tout, sur la théorisation explicative globale de la variation modale. Qu'il s'agisse de Gustave Guillaume, de Gérard Moignet ou de Joseph Hanse, pour l'époque de la première moitié du siècle précédent, ou de Robert Martin, pour la seconde, l'on a cherché à atteindre: "un principe unique ; ce principe, c'est la valeur modale du subjonctif» (Hanse 1960 : 5) ; de même selon Moignet : « il faut qu'il y ait entre tous les emplois du mode, quels qu'ils soient, un lien actuel, à la base de toutes les valeurs, un principe commun existant dans l'esprit» (Moignet 1959 t. I : 57).

\section{Le verbe proposer + complétive : aurait-il deux sens et une variation} modale?

Nous ne comptons bien évidemment pas résoudre, dans le cadre de cet article, le problème de l'interprétation de la valeur globale du subjonctif (si tant est qu'il y en ait une). Nous allons nous restreindre à une problématique bien circonscrite: l'emploi du mode subjonctif $v s$ mode indicatif en proposition complétive, lorsque celle-ci est régie par le verbe recteur proposer dans un contexte présumé explicatif et non-volitif, comme dans :

2 «L'approche statistique d'un problème grammatical exclut, nous le pensons, toute idée préconçue » (Nordahl $1969: 10)$.

3 Cette étude, projet de thèse de doctorat resté inachevé, a été publiée par les soins de Henri Bonnard.

$4 \quad$ «Pour une méthode immanente, une explication des emplois du subjonctif, s'il s'agit d'une étude synchronique, revient à une description des emplois du subjonctif : ceux-ci auront été expliqués quand on sera arrivé à décrire, aussi complètement que possible, les relations entre les morphèmes du subjonctif et les autres éléments de la langue » (Boysen $1971: 16$ ). 
(1) «Le constructivisme piagétien propose que, chez l'enfant, le nombre se construit par une synthèse logico-mathématique ». (Leroux 2005)

(2) «L'auteur propose que des sphéroïdes observés en fin de maladie puissent être l'agent de l'encéphalite, la protéine PrP SC. constituant la capside d'un acide nucléique inconnu. » (Institut de génétique et de microbiologie 2005)

En fait, il s'avère que, pour les dictionnaires tels que Le Robert, le T.L.F. et Littré, cet emploi ne semble pas exister; au mieux, on y trouve attesté le type proposer que $p$, mais uniquement en contexte volitif, où évidemment le subjonctif est obligatoire : «Il proposa que la motion fût mise aux voix immédiatement ». (Petit Robert 1996)

La majorité des dictionnaires de la langue contemporaine partent du sens fondamental de proposer, à savoir : «mettre devant (le regard, la perception) [...] [f]aire connaître à qqn, soumettre à son choix » (Le Robert) ; "présenter quelque chose à l'examen de quelqu'un (sans l'imposer)»(T.L.F.), sens étymologique qui, même s'il n'est pas exemplifié par les dictionnaires dans le contexte d'une complétive, est assez proche du sens de proposer que $p$ auquel nous nous intéresserons ici.

Les nombreuses grammaires, d'origine française ou non, que nous avons consultées ne traitent pas non plus du cas où proposer que $p$ aurait le sens d'un pur verbum dicendi, paraphrasable par avancer/émettre/faire l'hypothèse /supposer que p :

« Il se montra gentil et, pour les rassurer, émit l'hypothèse qu'il était arrivé un accident à leur fils ». ${ }^{5}$ (Aymé, cit. par Nordahl $1966: 157$ )

Quand le verbe proposer est listé dans les grammaires, il ne figure pas dans la série des verbes offrant un " choix de mode " tels que dire, sembler, ne pas croire que (Riegel, Pellat \& Rioul 2001); faire signe, faire comprendre, entendre... (Nordahl 1969).

Bien plus, une première sollicitation de l'avis de quelques locuteurs natifs (des collègues linguistes) laisse penser que le subjonctif serait tout à fait naturel, voire obligatoire pour l'emploi que nous avons appelé explicatif et non-volitif de proposer que $p$. L'influence du type volitif serait donc telle, si cette opinion reflète la réalité linguistique, que le subjonctif s'imposerait comme une servitude grammaticale (Gougenheim 1938), ne laissant aucun espace pour une différenciation sémantique marquée par le verbe (comme

5 Dans la Comprehensive Grammar of the English Language (Quirk et al. 1985 : $\S 16.31)$, une construction verbale anglaise correspondant à émettre l'hypothèse que serait étiquetée "factual verbs » (du sous-type «public type »), c'est-à-dire des verbes introduisant des actes assertifs indirects. 
c'est le cas pour, par exemple, la variation modale des verbes exemplifiés cidessus, ainsi que des propositions relatives).

Le type sémantique proposer que $p$, en contexte non-volitif, et son éventuelle variation modale soulèvent donc un certain nombre de questions. S'agit-il d'un emploi récent ou ancien ? Si cet emploi est récent, peut-on y voir une influence de l'anglais (où le type est fréquentissime dans les écrits scientifiques: $X$ proposes that $y$ is due to the factors $z, y$ and $w)$ ? Les théories existantes de la variation modale permettent-elles de décrire de manière satisfaisante l'emploi de proposer que $p$ en contexte volitif $v$ s nonvolitif? Une éventuelle variation modale en contexte non-volitif est-elle à décrire comme une variation libre?

Nous souhaitons donc confronter la réalité linguistique (telle qu'elle se reflète dans un large corpus d'exemples authentiques ${ }^{6}$ ) et certaines des théories explicatives globales, classiques et récentes, de la variation modale. Nous commencerons par un panorama des traitements de l'opposition modale dans différents travaux antérieurs, aussi bien des grammaires (françaises et scandinaves) que des ouvrages (ou articles) de nature plus théorique.

\section{Traitements antérieurs de l'opposition modale indicatif $v s$ subjonctif 3.1. Les grammaires de tradition française : quelques exemples}

Comme annoncé dans nos propos introductifs, de nombreux grammairiens et linguistes français cherchent à mettre en avant une théorie explicative globale de la variation modale, un principe unitaire aussi généralisant que possible. Certes, la terminologie varie d'une grammaire à l'autre, mais on retrouve des éléments communs aux différentes explications.

Chez Damourette \& Pichon (1936), on retient deux distinctions fondamentales: alors que l'indicatif serait le mode du «jugement», le subjonctif marquerait le «non-jugement» (cf. plus tard la distinction pragmatique entre "assertion» et "non-assertion»); deuxièmement, la distinction entre l'emploi «protagonistique » et l'emploi «locutoral» du subjonctif, selon que le responsable du non-jugement est le protagoniste ou le locuteur (cf. les théories de la polyphonie linguistique de Ducrot et Nølke).

Dans des grammaires plus récentes, le rapport de dépendance d'une action vis-à-vis d'une attitude de pensée du sujet parlant sera appelé rapport de "dépendance mentale» chez Charaudeau (1992: 484-492) alors que Denis \& Sancier-Chateau (1994: 481-494) parlent, à la suite de Robert Martin (1983, 1987 ; voir ci-dessous), de « mondes possibles » et d' « univers de croyance » (ou de "pesée critique »), tandis que Riegel, Pellat \& Rioul (2001 : 320-330) utilisent le terme d'«acte psychique» qui retiendrait l'aboutissement du procès. Ces grammaires, comme tant d'autres, soulignent

$6 \quad$ Les exemples sont tirés de la base textuelle Frantext et de Google. 
qu'avec le mode subjonctif, un grand principe est en jeu : l'« interprétation » prime sur l'actualisation du procès ${ }^{7}$.

Il n'est fait, nulle part dans ces grammaires, mention du cas qui nous intéresse, à savoir le verbe proposer + complétive en contexte non-volitif - à l'exception de quelques passages où sont listés des verbes de sens proche ou apparenté.

Pour Grevisse (1986), la valeur fondamentale du subjonctif consiste à indiquer que le locuteur/scripteur ne s'engage pas sur la réalité du fait $(\S 864$, $\S 1072)$; il serait donc, en termes plus généraux, un marqueur épistémique. Puis, dans un paragraphe qui nous intéresse plus particulièrement ( $\$ 1073 \mathrm{~b}$ ), l'on indique que certains verbes comme admettre, mettre (au sens de "supposer »), comprendre, concevoir, supposer peuvent entrainer le subjonctif ou l'indicatif sans différence de sens notable. Proposer, par contre, n'est pas répertorié dans cette catégorie.

\subsection{D'autres études spécialisées ou synthétisantes}

\subsubsection{Guillaume (1929)}

De Gustave Guillaume - nous n'entrerons pas ici dans les fondements de sa théorie générale des cinétismes et de la chronogenèse -, nous retiendrons son schéma (fig. 1 ci-après) figurant les trois modes, l'infinitif (in posse), le subjonctif (in fieri) et l'indicatif (in esse). Ainsi, selon Guillaume (Guillaume 1929: 31), «[1]a représentation indicative serait une représentation plus achevée, plus réalisée que la représentation subjonctive ».

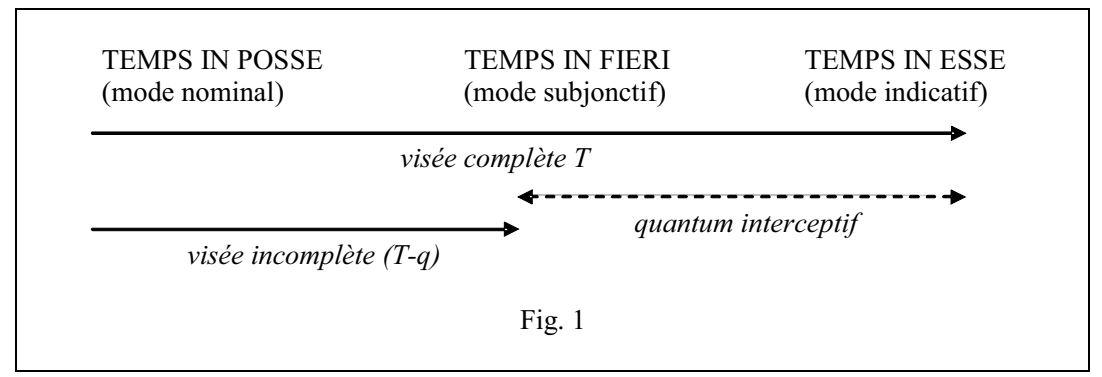

Nous nous demandons comment il conviendrait de classer proposer que $p$ en contexte non-volitif. L'idée que renferme ce verbe permet-elle la traversée complète ou seulement partielle du temps chronogénétique ? C'est une question à laquelle il nous semble difficile d'apporter une réponse ferme.

cf. aussi Wagner \& Pinchon (1962 : § 371). 


\subsubsection{Martin (1983)}

Parmi les linguistes qui affectionnent la quête d'un principe explicatif unitaire, mentionnons Robert Martin. Comme on sait, ce linguiste a proposé en 1983 sa sémantique fondée entre autres sur les deux notions logicoépistémiques de «mondes possibles» et d' « univers de croyance ». Dans sa conception sémantico-logique du subjonctif, «[1]e subjonctif est le mode qui marque l'appartenance non pas au monde $m_{0}$ de ce qui est, mais aux mondes possibles $m$, étant entendu [...] que l'inscription dans $m$ se fait par le biais de que et de sa fonction suspensive. » $(1983: 110)$

« [C]e morphème [sc que] a essentiellement pour fonction de suspendre la valeur de vérité de la proposition qu'il introduit et de la faire dépendre de

l'élément verbal ou conjonctionnel qui précède ». (ibid. : 106-107)

Il est hors de doute que, par ce modèle, Robert Martin parvient à rendre compte de façon élégante de plusieurs cas où apparaît le subjonctif, notamment en complétive. Cependant, dans le cas qui nous occupe, sa valeur explicative reste à examiner - et c'est ce que nous chercherons à faire dans cette étude. Constatons enfin que Robert Martin lui-même nous met en garde contre une croyance trop rigide en des lois « régissant » la variation modale (cf. l'exergue de la page 1).

\subsubsection{Nølke (1985)}

Dans son article de Langages intitulé «Le subjonctif. Fragments d'une théorie énonciative », Nølke (1985:55) cherche à expliquer certains emplois $\mathrm{du}$ subjonctif dans un cadre pragmatique. Ce chercheur suggère une corrélation entre le subjonctif et la notion de «polyphonie » et exprime ainsi l'idée centrale de son projet : «[1]e subjonctif marquerait une forme spéciale de polyphonie (au sens de Ducrot), ce mode étant ainsi une trace syntaxique de l'énonciation ». Nølke (ibid. : 55) ne considère pas que son approche contredise la théorie logico-sémantique (celle de Martin en particulier), mais plutôt que les deux se complètent mutuellement. Une notion avancée par Nølke nous semble prometteuse pour notre projet, celle de "polyphonie interne rapportée », par laquelle dans un exemple comme « Mais au fait, d'où tires-tu cet argent, puisque ce n'est pas vrai que ton père soit un riche planteur » (ibid. : 62), le locuteur associe les énonciateurs respectivement de ce n'est pas vrai et de la complétive à deux «figures" différentes. Par opposition à l'emploi de l'indicatif dans ce même énoncé («Mais au fait, d'où tires-tu cet argent, puisque ce n'est pas vrai que ton père est un riche planteur » (ibid. : 57)), le subjonctif introduit une nuance de doute, portant sur l'interprétation du locuteur de la pensée de l'autre. Dans sa conclusion, Nølke insiste sur la nécessité de « repérer les différents facteurs qui exercent 
leur influence » sur l'emploi de tel ou tel mode ; plutôt que de chercher des règles « exhaustives » dans une théorie unitaire, mieux vaut donc travailler au cas par cas en essayant de mesurer, dans chaque énoncé, le poids de tel ou tel élément explicatif.

D'autres grammairiens et linguistes insistent sur la difficulté d'attribuer une valeur unique au mode complexe qu'est le subjonctif. Mentionnons, à ce sujet, les travaux d'Imbs (1953), de Cohen (1961) et ceux du Norvégien Nordahl (1969 : 15-16), qui, après avoir présenté les théories explicatives de son époque de manière très détaillée (Théorie amodale et afonctionnelle; Théorie amodale et fonctionnelle; Théorie temporelle; Théorie modale I: réalité/non réalité et Théorie modale II : objectivité/subjectivité) se contente d'une répartition de ses 24000 occurrences de complétives dans trois systèmes : le volitif, le subjectif et le dubitatif (1969 : 249). Enfin, citons les propos de Yaguello (2003: 176), dans Le Grand livre de la langue française :

«Le subjonctif exprime d'une part le non-certain, l'hypothétique, le peu probable (y compris la négation du certain) et s'oppose à l'indicatif, associé au certain et au probable. Mais par ailleurs, il constitue la marque grammaticale de la modalité appréciative, ce qui peut paraître paradoxal puisque cette modalité s'articule sur du factif; elle est donc présupposante : je regrette, je me réjouis [...] j'apprécie ...que Lionel soit parti présuppose «Lionel est parti ». Le subjonctif est également requis dans les propositions complétives régies par des verbes déontiques ou de volonté (modalité intersubjective) : Je veux, je souhaite [...] que Lionel parte. On ne saurait donc attribuer au subjonctif une valeur claire et constante $[\ldots] »$.

\section{Brève excursion diachronique}

Nous l'avons dit ci-dessus, aucun des dictionnaires de langue moderne ne mentionne le sens de proposer explicatif et non-volitif. Mais quels sens de proposer sont attestés dans le Dictionnaire historique de la langue francaise d'Alain Rey?

«Proposer est emprunté avec francisation d'après poser (v 1120) au latin proponere, de pro « devant » [...] et ponere «placer» [...]. Ce verbe signifie proprement «placer devant les yeux, présenter» et, au figuré «(se) représenter mentalement», "faire un exposé, annoncer», "offrir (une récompense, un sujet de discussion) » et « se donner pour but, dessein de ».

Le verbe a été repris au sens propre, «présenter au regard», le plus souvent avec la notion seconde de "donner comme modèle, comme exemple», en parlant de Dieu. Il a bientôt repris du latin le sens de «projeter, avoir l'intention de » (1130-1140) dans la construction indirecte $[\ldots] »$. 
Le sens figuré du verbe latin proponere de «faire un exposé, annoncer » dont il est fait mention ici semble bien correspondre au proposer verbum dicendi, paraphrasable par avancer ou émettre l'idée que, qui nous intéresse. Il est à noter, cependant, que Rey ne donne aucun exemple avec complétive.

Dans le Dictionnaire de l'ancienne langue française de Godefroy (1889 : 439), en revanche, l'on trouve un exemple de proposer que dans le sens de «avancer, soutenir, exposer que $\mathrm{p} »$ :

«Car tu avois proposé que l'agriculture est le plus facile art du monde » (La Boetie, Mesnag. De Xeuoph., Feugère)

De même, on trouve dans le Dictionnaire du moyen français (A.T.I.L.F.), sous la rubrique «Proposer que. 'Exposer, avancer, soutenir que' », d'autres occurrences de notre type :

(6) «ledit Corbeant avoit confessé ou proposé qu'il estoit clerc, combien que de la partie de l'evesque d'Arras feust dit qu'il avoit proposé qu'il estoit clerc non marié » (BAYE, I, 1400-1410, 38)

«Ce jour, vint en la Court le recteur et plusieurs des maistres de l'Université en leurs abitz acoustumés, et firent proposer par la bouche de maistre Guillaume Erard, maistre en theologie, qu'ilz venoient pour faire une grief complainte » (FAUQ., III, 1431-1435, 101)

Ajoutons un exemple du XVI ${ }^{e}$ siècle, issu de la base textuelle Frantext :

«Nostre Seigneur Jesus pour mieux inciter ces Apostres afin qu'ils s'esvertuent tant mieux à faire leur office, leur propose que le fruit de leur labeur est présent ». (CALVIN, Jean, Institution de la religion chrestienne, livre quatrième, 1560)

Cependant, les recherches que nous avons effectuées sur Frantext nous permettent d'observer que les quelques exemples attestant l'emploi de proposer que en tant que pur verbum dicendi, s'arrêtent approximativement au XVII' ${ }^{\mathrm{e}}$ siècle. En voici à titre d'illustration un exemple de 1610 :

(9) «Veu que si ceste opinion estoit fondee sur la verité, il faudroit par necessité conclure une chose des plus absurdes et fabuleuses que les hommes ayent jamais pensees : par ce qu'en proposant que les françois n'ont rien dict que les italiens et les latins n'ayent dict auparavant». (DEIMIER, Pierre de, L'Académie de l'art poétique, où sont vivement esclaircis et déduicts les moyens par où l'on peut parvenir à la vraye et parfaite connoissance de la poésie françoise, 1610)

Il ne s'agit donc pas d'un emploi tout à fait récent ; tout au plus pourrait-on parler d'un abandon de proposer dans ce sens, pendant une longue période. 
Faut-il voir dans ce retour une influence moderne de l'anglais ${ }^{8}$, ce qui n'est pas totalement inconcevable, compte tenu de l'importance de l'anglais dans les écrits scientifiques et du fait que de nombreux chercheurs lisent autant de travaux scientifiques en anglais que dans leur propre langue? Il s'agirait donc, selon cette hypothèse, d'une réapparition par emprunt sémantique, emprunt qui ne serait pas encore entré dans les dictionnaires français. Illustrons cette hypothèse à l'aide de l'exemple suivant, tiré du résumé de la thèse The New Civil Code of the Russian Federation and Private International Law, de Viktor P. Zvekov (1999) :

(10) «On the other hand, there is a movement for an overarching set of principles on private international law to be consolidated within the C.C.R.F. The author proposes that both can be done : general principles can be expressed in the C.C.R.F. while legislation in specific areas could have their own rules on private international law. »

[...]

D'autre part, il existe un mouvement désirant intégrer au sein du Code une série de principes notoires de droit international privé. L'auteur propose que les deux avenues sont réalisables: les principes généraux peuvent être formulés dans le Code, alors que la législation régissant des domaines spécialisés pourrait avoir ses propres règles de droit international privé.

\section{Variation modale en synchronie : le témoignage du corpus 5.1. Corpus : méthode de prélèvement et genre textuel}

Les exemples analysés dans cette étude, notre corpus de travail, ont été relevés dans un large corpus exploratoire : la base textuelle Frantext et le moteur de recherche Google. Ce corpus de travail comprend une trentaine d'exemples de proposer + complétive en contexte non-volitif. Pour les obtenir, nous avons effectué une recherche sur Google, en donnant quelques éléments de contexte. Voici quelques exemples de séquences rentrées dans le moteur de recherche :

\footnotetext{
"dans son article * propose que"

"en conclusion * propose que"

"l'auteur propose que"
}

Ensuite, nous avons sélectionné les cas de proposer + complétive nous semblant relever, au premier abord, du sens non-volitif, écartant ainsi

$8 \quad$ Voici la définition de Longman : «propose : [...] * THEORY * To suggest an idea, method etc as an answer to a scientific question or as a better way of doing something. » 
plusieurs dizaines d'occurrences de proposer en contexte volitif. Cette première sélection a déjà révélé qu'il n'est pas simple de séparer nettement les exemples relevant du type volitif vs non-volitif.

Observons tout d'abord que les exemples obtenus, pour l'essentiel, sont extraits de textes à caractère scientifique, ce qui n'est pas surprenant compte tenu du sens de proposer que, qui, ici, signifie à peu près «avancer une hypothèse ». Ainsi, les textes dépouillés appartiennent à un genre discursif bien restreint: résumés de thèses et/ou comptes rendus d'ouvrages scientifiques.

Enfin, rappelons que nous avons augmenté notre matériau d'observation en consultant la base textuelle Frantext. Celle-ci nous a fourni quelques exemples de proposer que en contexte non-volitif, issus de textes plus anciens (voir supra). Ces exemples ne seront pas pris en compte dans l'analyse étant donné que nous chercherons plutôt, ici, à mener une analyse en synchronie.

\subsection{Fréquence}

Sur les 35 extraits de textes de proposer que $p$ (au sens non-volitif) de notre corpus :

- 7 exemples comportent le subjonctif présent ou passé dans la complétive ;

- 20 exemples comportent l'indicatif présent, passé ou futur dans la complétive $^{9}$;

- 5 exemples comportent un conditionnel présent ou passé dans la complétive ;

- pour les 4 derniers exemples, il est impossible, compte tenu de la forme verbale utilisée, de trancher sur le mode employé.

Il y a donc bien variation modale; notons toutefois que les emplois à l'indicatif sont en majorité assez nette.

\section{Y a-t-il un principe explicatif unitaire ? Quelques éléments de réponses préliminaires}

Question fondamentale chapeautant toutes les autres: certaines théories existantes de la variation modale permettent-elles de décrire de manière satisfaisante l'emploi de proposer que $p$ en contexte volitif $v s$ non-volitif?

9 Un des extraits de notre corpus compte une occurrence de proposer régissant deux complétives, l'une au subjonctif, l'autre à l'indicatif. Nous avons donc comptabilisé cet extrait deux fois : une fois sous la catégorie des exemples comportant le subjonctif, une fois sous les exemples comportant l'indicatif. 
Une éventuelle variation modale en contexte non-volitif est-elle à décrire comme une variation libre?

Compte tenu de la taille du recueil d'occurrences, il nous est difficile de nous prononcer sur la question de savoir s'il s'agit d'une variation «libre » ou bien si cette variation est significative. Essayons à présent de confronter nos exemples à quatre théories sur la variation modale.

\subsection{Vision de " réalisation effective » vs vision de «non-réalisation » (ou de réalisation potentielle) ${ }^{10}$}

Selon cette hypothèse, le subjonctif apparaît lorsque le procès de la complétive est envisagé de manière "prospective » par le locuteur - la réalisation est alors «potentielle»-, tandis que la vision de « rétrospectivité » ou de réalisation « effective » (Charaudeau 1992) implique l'indicatif, qui indique alors que le procès est/a été actualisé. Le subjonctif constituerait alors, lorsqu'il apparaît dans des contextes à première vue nonvolitifs, une trace - ne serait-ce que sous une forme très atténuée - du sens volitif qui viendrait s'ajouter à la modalité de «constat». Cette hypothèse explicative pourrait s'appliquer aux exemples suivants :

(11) «Dans son article, elle propose que le concept de justice comprenne deux éléments fondamentaux, soit l'avis ou le fait que les personnes doivent être jugées seulement sur des faits dont elles ont connaissance et alors qu'elles ont l'occasion de faire valoir leur point de vue et l'égalité de tous devant la justice, sans égard à la race, sexe, nationalité d'origine... ».

«En outre, il montre que les intuitions sémantiques concernant la relation entre le verbe et son CO s'appliquent à une large gamme d'items lexicaux. Aussi, comme 'cas' et ' $\theta$-rôle' n'étaient pas distinguées en raison de la prédominance de l'analyse de Fillmore (1968) 'Case for Case' il propose que $\mathrm{CO}$ reçoive un cas factif ainsi défini : 'the case of the object or being resulting from the action or state identified by the verb, or understood as part of the meaning of the verb'. »

où proposer impliquerait non seulement le fait que l'auteur asserte/pose un certain propos, mais aussi qu'il ou elle soumet celui-ci à l'appréciation/au jugement de ses interlocuteurs.

10 Il existe différents avatars de cette théorie : in fieri vs in esse ; « monde de ce qui est»vs " monde possible »; rétrospectivité vs prospectivité, etc. Pour l'emploi de ces deux derniers termes, voir par exemple Wilmet (1998: 338 et $s q$.). Le « prospectif» est lié à la modalité optative : ordre, conseil, etc. Wilmet utilise également les termes « inactuel » (subjonctif) $v s ~ «$ actuel» (indicatif). 
S'agissant de l'hypothèse de réalisation "potentielle »vs «effective » («prospectivité »vs « rétrospectivité »), il existe pourtant, dans notre corpus, un exemple «déconcertant » :

«En se basant sur les caractéristiques géologiques et géochimiques de ces trois dépôts aurifères, l'auteur propose que ceux-ci aient été contrôlés par la structure de la zone de cisaillement de Wulong, et que la minéralisation d'or est génétiquement reliée avec l'intrusion de Sanguliu. »

Ici, le subjonctif (portant sur un événement passé et donc déjà actualisé) paraît surprenant. Le deuxième verbe de la complétive est à l'indicatif, ce qui annule plus ou moins toute tentative d'explication du mode du premier verbe de la complétive. C'est là une constatation qui nous amène à la conclusion que l'hypothèse en question, en ce qui concerne notre type d'emploi, n'est guère satisfaisante. Il semble bien difficile de défendre, pour (13), l'idée d'une différence significative de sens entre la forme aient été et est.

\subsection{L'hypothèse de la « contamination »}

$\mathrm{Au}$ vu de cas comme (11) et (12), il n'est pas trop difficile d'imaginer un effet de contamination analogique ou "par automatisme »: là où l'intention locutorale doit être décrite comme aussi bien prospective que rétrospective, voire indécidable, ce sera le mode pour ainsi dire «normal » de proposer que $p$, le subjonctif, qui se présente, étant donné que l'emploi nettement volitif de proposer que $p$ est largement prédominant.

\section{3. «Interprétation » vs « constat/déclaration »; « subjectivité » vs « objectivité »}

Cette hypothèse, également classique, semble bien incapable de rendre compte de notre variation modale: dans des contextes scientifiques explicatifs, on trouve une alternance entre le subjonctif (minoritaire) et l'indicatif (majoritaire) qui ne s'explique guère par une variation en « interprétativité ».

Une autre hypothèse est que la distinction subjectivité/objectivité recouvre l'opposition modale subjonctif/indicatif. Comparons les exemples suivants, l'un au subjonctif, l'autre à l'indicatif:

(14) «L'auteur propose que des sphéroïdes observés en fin de maladie puissent être l'agent de l'encéphalite, la protéine PrP SC. constituant la capside d'un acide nucléique inconnu. »

(15) «L'auteur propose que les comportements alcooliques observés en milieu autochtone sont révélateurs d'une trame identitaire complexe qui, en 
certains lieux, ouvre sur des espaces pouvant être qualifiés de "meurtriers". »

Dans ce contexte, on pourrait soutenir, à l'instar de Confais (1990:242), que le subjonctif sert à "désamorcer le potentiel déclaratif» de la séquence concernée. Ici aussi, il faudrait parler d' "effets d'objectivité » $v s$ «de subjectivité » avec des degrés plus ou moins forts. Cependant, l'hypothèse subjectivité/objectivité présente un double problème. Tout d'abord, les notions de subjectivité et d'objectivité sont des notions préthéoriques. Ensuite, l'hypothèse est non-vérifiable (non-testable, non-réfutable).

\subsection{Polyphonie interne / polyphonie externe (DR)}

Pour ce qui est de l'hypothèse polyphonique, l'observation faite par Nølke concernant la «polyphonie interne rapportée » nous semble intéressante afin de rendre compte de la variation modale des exemples de notre propre corpus. Rappelons l'hypothèse de Nølke (1985: 61) : « [1]e subjonctif est un marqueur syntaxique de polyphonie interne au sens strict [...]. » Le locuteur associe les énonciateurs de la principale et de la complétive à deux « figures » différentes (Nølke 1985 : 63). Proposons trois exemples de notre corpus :

(16) «À partir de deux illustrations cliniques, l'auteur propose que, en raison de la nature plurielle dès l'origine du psychisme humain, il n'y ait pas dans cette double direction de véritable incompatibilité ».

(17) «De manière générale, Siegler propose que dans l'acquisition des faits arithmétiques, la maturation des stratégies ne suit pas une logique stricte en étapes. »

«Pour expliquer certaines de ces contradictions, l'auteur propose que les effets de l'éducation peuvent être différents selon le groupe cible de stigmate (les Juifs, les Noirs) puisque ces groupes ne sont pas victimes des mêmes stéréotypes. »

En (17) et (18), l'on aurait ainsi affaire à de purs discours rapportés, entraînant une polyphonie «externe » et «objective» (ou présentée par le locuteur de manière extérieure) : les locuteurs des énoncés (17) et (18), avec l'indicatif, n'expriment ni association ni distance vis-à-vis du contenu de la complétive. En (16) au contraire, on décèlerait sinon un doute du moins une dissociation de la part du locuteur-rapporteur vis-à-vis du contenu de la proposition complétive.

Selon cette hypothèse, on devrait donc considérer que cette dissociation est un « reflet subjectif », dans la mesure où dans le cas où proposer que est suivi du subjonctif, on pourrait y voir la « volonté » du locuteur-rapporteur de ne pas se contenter de faire un rapport «objectif» de la proposition de la 
complétive, mais plutôt de faire une « intrusion » dans le discours cité pour mettre en avant sa propre interprétation, vis-à-vis de celle de l'énonciateur. Cependant, cette hypothèse, comme celle sous 6.3, est difficilement démontrable.

Remarquons, à propos de l'hypothèse polyphonique, l'existence d'un parallélisme entre l'interprétation de la variation modale et celle du conditionnel, comme l'illustrent les exemples suivants :

(19) «Enfin, les études comportant le plus grand nombre de cas n'ont pas observé d'association. Sans en exclure un, l'auteur propose que ce polymorphisme ne devrait pas avoir d'impact significatif sur la prééclampsie. »

«Ses réactions ont été effectuées avec des quantités équimolaires de méthyllithium et de vinylalane dans l'heptane. L'auteur propose que l'espèce réactive ne serait pas le trialkylvinylalanate de lithium 67 , mais bien le vinyllithium 68 obtenu par dissociation du trialkylvinylalanate de lithium 67 (schéma 30). »

Il faut à notre sens comprendre le devrait de (19) comme un conditionnel «d'énonciateur » ${ }^{11}$, qui donc est « repris » tel quel du discours direct soustendant le discours rapporté :

(19') «Je propose que ce polymorphisme ne devrait pas avoir d'impact significatif...»

tandis que le serait de (20) est à comprendre comme une médiation épistémique (Kronning ibid.), c'est-à-dire une marque de distanciation de la part du locuteur, correspondant par conséquent à un indicatif en discours direct :

« Je propose que l'espèce réactive n'est pas le trialkylvinylalanate... »

Ainsi, l'opposition entre subjonctif $v s$ indicatif dans la théorie de la polyphonie correspondrait aux deux interprétations du conditionnel - en médiation énonciative vs médiation épistémique.

Il est également intéressant d'examiner l'exemple (21) :

(21) «En conclusion je propose que les études sur la distribution différentielle des maladies ne devraient pas s'appuyer uniquement sur des différences de statut socio-économique. Ces études devraient aussi examiner comment les pratiques sociales des individus sont liées aux ressources matérielles. De plus, je conclus que les variables qui caractérisent des attributs individuels

11 C'est-à-dire comme un «conditionnel épistémique modalisant» (Kronning 2005, 2007). 
participent au même processus que les variables qui caractérisent des attributs collectifs; conjointement ces deux types de variables façonnent le phénomène maintenant connu sous le vocable de production sociale de la maladie. »"

Cela nous semble être un cas de ce que Kronning $(2005,2007)$ appelle " modalisation complexe », dans la mesure où ce devraient exprime, à la base d'un raisonnement - «En conclusion...» - quelque chose vu comme nécessairement vrai.

\section{En guise de conclusion provisoire}

\subsection{Du côté des données}

Tout d'abord, le sens non-volitif du verbe proposer + complétive n'est décrit ni dans les grammaires ni dans les dictionnaires modernes. Il semble cependant avoir existé dans des époques antérieures, au moins jusqu'au XVII ${ }^{\mathrm{e}}$ siècle. Par ailleurs, dans la mesure où cet emploi apparaît actuellement, il semble bien y avoir une variation modale. Nos dépouillements préliminaires indiquent que l'indicatif est clairement majoritaire.

\subsection{Du côté de la théorie}

Même si la plupart des théories explicatives «globales » semblent pouvoir rendre compte de façon élégante de bon nombre de cas de la variation modale, elles restent toutes non-démontrables dans le sens scientifique : les preuves indépendantes et indiscutables font défaut.

Ainsi, jusqu'à nouvel ordre, la seule hypothèse globale non-falsifiée (le principe poppérien!) reste celle de la variation libre, ou stylistique : il semble bien exister, à l'époque actuelle et dans le genre discursif examiné, une hésitation quant à l'emploi modal dans le cas de proposer que $p$ dans des contextes non-volitifs. L'emploi du subjonctif, qui est très minoritaire dans le genre textuel des exemples analysés, semble au mieux s'expliquer par une influence des emplois clairement volitifs, emplois de loin les plus fréquents.

\section{Références}

Boysen, G. (1971). Subjonctif et hiérarchie, Odense : University Press.

Börjesson, L. (1966). La fréquence du subjonctif dans les subordonnées complétives introduites par que, Studia Neophilologica 38 : 3-64.

Charaudeau, P. (1992). Grammaire du sens et de l'expression, Paris: Hachette Éducation.

Cohen, M. (1961). Le subjonctif en français contemporain. Tableau documentaire, Paris : SDEDES. 
Confais, J.-P. (1990). Temps, mode, aspect, Toulouse : Presses Universitaires du Mirail.

Damourette, J. et Pichon, E. (1936). Des mots à la pensée. Essai de grammaire de la langue française. Tome cinquième. Verbe (fin). Auxiliaires, temps, modes, voix, Paris : d'Artrey.

Denis, D. et Sancier-Chateau, A. (1994). Grammaire du français, Paris : Le Livre de poche.

Dictionnaire du moyen français, http://www.atilf.fr/blmf/.

Godefroy, F. (1889) (troisième réimpression de 1969). Dictionnaire de l'ancienne langue francaise, Nendeln/Liechtenstein : Kraus Reprint.

Gougenheim, G. (1938). Système grammatical de la langue française, Paris : Bibliothèque du français moderne.

Grevisse, M. (1986) (12 ${ }^{\text {ème }}$ éd.). Le Bon usage, Paris-Gembloux : Duculot.

Guillaume, G. (1929) (réédition de 1993). Temps et verbe. Théorie des aspects, des modes et des temps, Paris : Honoré Champion.

Hanse, J. (1960). La valeur modale du subjonctif, Bruxelles.

Imbs, P. (1953). Le subjonctif en français moderne. Essai de grammaire descriptive, Paris : Publications de la Faculté des Lettres Strasbourg.

Kronning, H. (2005). Polyphonie, médiation et modalisation: le cas du conditionnel épistémique, in: J. Bres, et al., (éds), Dialogisme, polyphonie: approches linguistiques. Bruxelles: De Boeck-Duculot: 297-312.

Kronning, H. (2007). Om epistemiska uttryck i de romanska språken, in : Kungl. Vetenskaps-Societeten i Uppsala. Arsbok 2006, Uppsala : Kungl. Vetenskaps-Societeten [Royal Society of Sciences] : 107-141.

Littré, E. (1877). Dictionnaire de la langue française, Paris : Hachette.

Longman Dictionary of Contemporary English (1995), Harlow : Longman Dictionaries

Martin, R. (1983). Pour une logique du sens, Paris : P.U.F.

Martin, R. (1987). Langage et croyance, Bruxelles : Mardaga.

Moignet, G. (1959). Essai sur le mode subjonctif en latin postclassique et en ancien français, Tome I. Paris : P.U.F.

Nølke, H. (1985). Le subjonctif, fragments d'une théorie énonciative, Langages $80: 55-70$.

Nordahl, H. (1966). Les systèmes du subjonctif corrélatif. Étude sur l'emploi des modes dans la subordonnée complétive en français moderne, Bergen/Oslo : Universitetsforlaget.

Quirk, R., Greenbaum, S., Leech, G. \& Svartvik, J. (1985). A Comprehensive Grammar of the English Language, Longman.

Riegel, M.; Pellat, J.-C. ; Rioul, R. (2001). Grammaire méthodique du français, Paris : P.U.F.

Robert, P. (1996). Le Nouveau Petit Robert. Dictionnaire alphabétique et analogique de la langue française, Paris : Dictionnaires Le Robert. 
Togeby, K. (1982). Grammaire française, vol. V, Copenhague : Akademisk Forlag.

Trésor de la langue française informatisé, http://atilf.atilf.fr/tlf.htm.

Wagner, R.L. et Pinchon, J . (1962). Grammaire du français classique et moderne, Paris : Hachette.

Wilmet, M. (1998). Grammaire critique du français, Paris: HachetteDuculot.

Yaguello, M. (sous la direction de). (2003). Le grand livre de la langue française, Paris : Seuil. 